\title{
MIS Fusion of the SI Joint: Does Prior Lumbar Spinal Fusion Affect Patient Outcomes?
}

\author{
Leonard Rudolf*
}

\section{Mascoma Street, Lebanon, NH 03766, USA}

\begin{abstract}
Background: Sacroiliac (SI) joint pain is a challenging condition to manage as it can mimic discogenic or radicular low back pain, and present as low back, hip, groin and/or buttock pain. Patients may present with a combination of lumbar spine and SI joint symptoms, further complicating the diagnosis and treatment algorithm [1-3]. SI joint pain after lumbar spinal fusion has been reported in the literature. Both clinical and biomechanical studies show the SI joint to be susceptible to increased motion and stress at the articular surface with up to $40-75 \%$ of patients developing significant SI joint degeneration after 5 years.
\end{abstract}

In a recent case series study of 50 patients who underwent minimally invasive SI joint arthrodesis, $50 \%$ had undergone previous lumbar spinal fusion and $18 \%$ had symptomatic lumbar spine pathology treated conservatively [4].

The purpose of this study is to determine if history of previous lumbar fusion or lumbar pathology affects patient outcomes after MIS SI joint fusion surgery.

Methods: We report on 40 patients with 24 month follow up treated with MIS SI joint fusion using a series of triangular porous plasma coated titanium implants (iFuse, SI-Bone, Inc. San Jose, CA). Outcomes using a numerical rating scale (NRS) for pain were obtained at 3-, 6-, 12- and 24 month follow up intervals. Additionally, patient satisfaction was collected at the latest follow up interval. Patients were separated into 3 cohorts: 1) underwent prior lumbar spine fusion $(\mathrm{PF}), 2$ ) no history of previous lumbar spine fusion (NF), 3) no history of previous lumbar spine fusion with symptomatic lumbar spine pathology treated conservatively (LP). A repeated measures analysis of variance (rANOVA) was used to determine if the change in NRS pain scores differed across timepoints and subgroups. A decrease in NRS by 2 points was deemed clinically significant [5].

Results: Mean age was $54( \pm 13)$ years and varied slightly but not statistically between groups. All subgroups experienced a clinically and statistically significant reduction in pain at all time points (mean change $>2$ points, $\mathrm{p}<0.001$ ). There was a statistically significant effect of cohort ( $\mathrm{p}=0.045)$, with the NF cohort (no prior lumbar spinal fusion) having a somewhat greater decrease in pain (by approximately 1 point) compared to the other 2 groups (PF and LP).Patient reported satisfaction by cohort was: $89 \%(\mathrm{NF}), 92 \%(\mathrm{PF})$ and $63 \%(\mathrm{LP})$. Overall satisfaction rate was $87 \%$.

Discussion and Conclusion: Patients with SI joint pain, regardless of prior lumbar spine fusion history, show significant improvement in pain after minimally invasive SI joint fusion. The presence of symptomatic lumbar spine pathology potentially confounds the treatment affect, as patients may not be able to discriminate between symptoms arising from the SI joint and the lumbar spine. These patients expressed a lower satisfaction with surgery. Patients without other confounding lumbar spine pathology and who have not undergone previous spine surgery tend to be younger and experience a greater reduction in pain.

Keywords: Sacroiliac (SI) joint, lumbar fusion, minimally invasive surgery, MIS arthrodesis.

\section{INTRODUCTION}

Sacroiliac (SI) joint pain is a challenging condition to manage as it can mimic discogenic or radicular low back pain, and present as low back, hip, groin and/or buttock pain. Patients may present with a combination of lumbar spine and SI joint symptoms, further complicating the diagnosis and treatment algorithm. SI joint pain after lumbar spinal fusion has been widely reported in the literature. Both clinical and biomechanical studies show the SI joint to be susceptible to increased motion and stress at the articular surface after

*Address correspondence to this author at the 129 Mascoma Street, Lebanon, NH 03766, USA; Tel: 603448 6344; Fax: 603448 3405;

E-mail: leonardrudolf@gmail.com lumbar spinal fusion with up to $40-75 \%$ of patients developing significant SI joint degeneration after 5 years [6-8].

Despite the large number of patients with SI joint pain, treatment options have been limited to conservative care involving physical therapy and joint injections, or traditional open SI joint fusion surgery. Open arthrodesis procedures reported in the literature require relatively large incisions, significant bone harvesting, lengthy hospital stays and require the patient to be non-weight bearing for several months.

In our recent case series study of 50 patients who underwent minimally invasive SI joint arthrodesis, $50 \%$ of patients had undergone previous lumbar spinal fusion. Eighteen percent $(18 \%)$ of patients had concomitant 
symptomatic lumbar spine pathology that they chose to manage conservatively.

The purpose of this study is to determine if history of previous lumbar fusion or concomitant lumbar pathology affects patient outcomes after minimally invasive SI joint fusion surgery using a series of triangular porous plasma coated titanium implants.

\section{MATERIALS AND METHODS}

A review of the previously reported 50 patient cohort who underwent minimally invasive SI joint fusion revealed 45 patients available at the 2 year follow up interval. Out of these 45,1 patient had no pre-operative data and the remaining 4 patients underwent concomitant lumbar spine surgery and were thus excluded from this analysis, leaving a total of 40 patients available for the current study. The medical charts of these 40 patients were reviewed for perioperative metrics and pain scores using numerical rating scale (NRS) at 3, 6, 12 and 24 months postoperatively. In addition, patient satisfaction was collected at the 24 month time point. Subjects weredivided into 3 cohorts: 1 ) no history of previous lumbar spine fusion and no concomitant symptomatic lumbar spine pathology (NF), 2) underwent prior lumbar spinal fusion (PF), and3) no history of previous lumbar spine fusion with concomitant symptomatic lumbar spine pathology treated conservatively (LP). A repeated analysis of variance (ANOVA, PROC MIXED) was performed using SAS version 9.0 (Cary, NC) to evaluate change in pain across cohorts and post-operative time points. Clinical significance was defined as an improvement of 2 points from baseline [5]. Patient satisfaction is reported as percentage of patients who would undergo the same surgery for the same result.

\section{Procedure Description}

All subjects were diagnosed with either degenerative sacroiliitis or sacroiliac joint disruption using a combination of history, clinical exam, and positive diagnostic injection. All patients presented with chronic lower back pain refractory to prolonged (greater than 6 months) conservative care. The most common chief complaint was posterior pain located close to the SI joint. A thorough physical and clinical exam was performed on all patients, emphasizing the lumbar spine, SI joint and hip axis. Provocative physical examination maneuvers were used to guide subsequent diagnostic activities. All patients with suspected SI joint pain underwent imaging with X-ray, CT and/or MRI to evaluate SI joint pathology and exclude lumbar spine and hip pathology. When clinical, physical and radiographic examinations were concordant, patients were sent to a pain physician for confirmatory image-guided injections of the SI joint. A $75 \%$ reduction in pain, as measured on a visual analog scale, with at least $75 \%$ pain relief, immediately following injection of local anesthetic was used to confirm the SI joint as the pain generator $[8,9]$.

All patients underwent minimally invasive SI joint fusion using the iFuse Implant System (SI-BONE, INC, San Jose, $\mathrm{CA})$. The procedure involves the placement of 3 triangular, porous plasma coated, titanium implants across the sacroiliac joint (Fig. 1a, b). The objective of this surgery is to achieve arthrodesis through a permanent linkage across the joint, relying on bone in growth for permanent stabilization of the implant. Surgery is performed under general anesthesia with the patient in the prone position. Fluoroscopic guidance is used throughout the procedure to monitor implant placement.

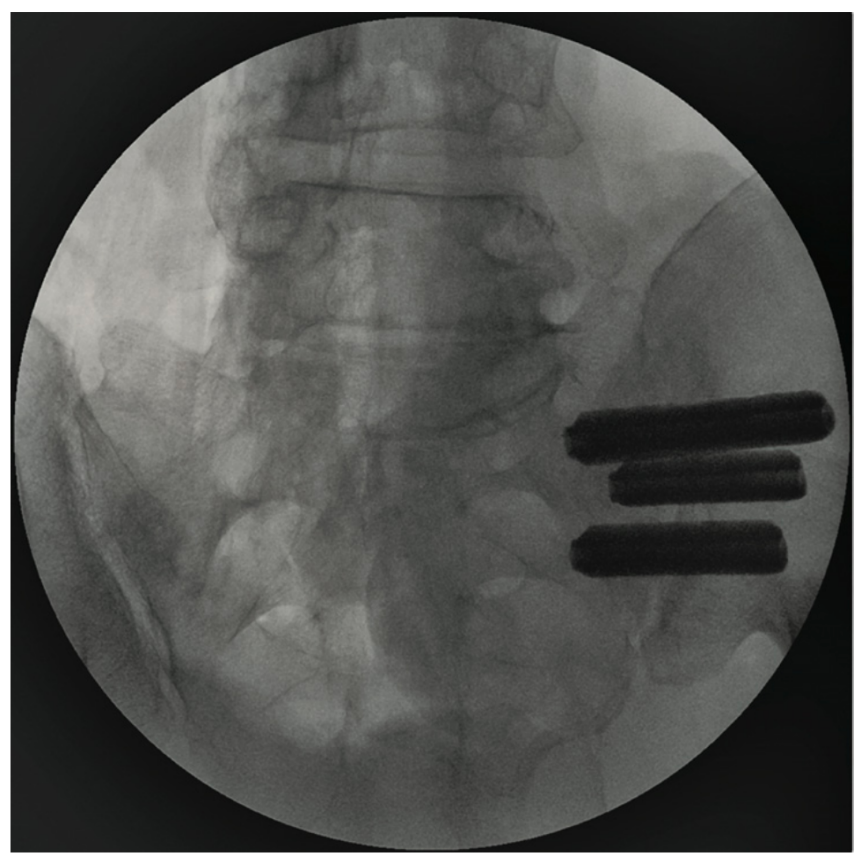

Fig. (1a). AP view of 3 implants in place

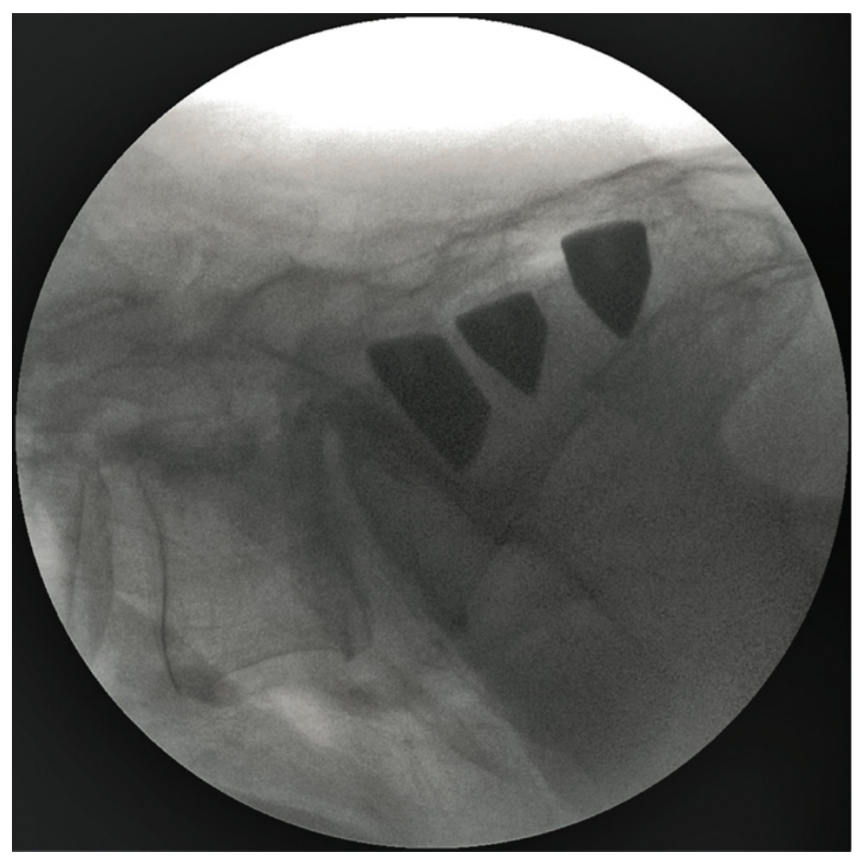

Fig. (1b). Lateral view of 3 implants in place.

\section{Technique Description}

A Steinman pin is centered halfway between the anterior cortex of the sacrum and the anterior border of the spinal canal, at least one centimeter distal to the S1 endplate. A soft tissue protector is the placed over the pin and a depth gage is used to determine the appropriate implant length. A drill is placed over the pin and advanced until the appropriate depth is reached, paying careful attention to avoid the sacral foramina and the anterior sacral cortex. The area is then 
broached over the pin to the identical depth. The implant is inserted over the pin with approximately $2-5 \mathrm{~mm}$ of the proximal side of the implant remaining outside of the cortex of the ilium. The parallel pin guide is then used to aid in the placement of the additional two implants. The first two implants are generally located above the S1 foramen. The third implant is typically placed between the S1 and S2 foramina.

\section{RESULTS}

We report on 40 patients with available 24 month follow up data treated with MIS SI joint fusion using a series of triangular porous plasma coated titanium implants. Fifteen patients underwent prior lumbar spinal surgery: 4 received single level lumbar fusion, 11 underwent multi-level lumbar spinal fusion (Table 3). The most common previous lumbar spinal surgery was fusion of L4 to the sacrum $(n=9)$. One patient underwent hip replacement surgery. Mean age at the time of surgery was $54( \pm 13)$ years. Mean age between groups varied slightly. Patients who had never undergone surgery were on average a decade younger than the other 2 cohorts. Mean age for the NF, PF and LP cohorts was 49, 58 and 58 years respectively (Table 1). Mean operating time was $62( \pm 18)$ minutes for all subjects and was slightly shorter in the NF cohort, although this difference was not statistically significant.

Outcomes using numerical rating scale (NRS) for pain were obtained at 3-, 6-, 12- and 24 month follow up (Table 2). Additionally, patient satisfaction was collected at the last follow up interval. All three cohorts experienced a clinically and highly statistically significant reduction in pain scores at all time points (rANOVA $\mathrm{p}<0.0001$ ) (Fig. 2). There was a statistically significant effect of cohort $(\mathrm{p}=0.045)$, with the NFcohort (no prior lumbar spinal fusion) having a somewhat greater decrease in pain (by approximately 1 point) compared to the other 2 groups (PF and LP). Analysis revealed that subjects in the NF cohort were on average younger and had overall lower pain scores by, on average 1.32 points, at all follow up intervals $(\mathrm{p}=0.0027$ ) (Fig. 3). This indicatesthat this cohort is in fact a separate population as compared to the other 2 cohorts. Patient reported satisfaction was: $89 \%$ for NF, $92 \%$ for PF and $63 \%$ in the LP cohort. Satisfaction for combined cohorts was $87 \%$.

\section{COMPLICATIONS}

There were 8peri-operative complications in 7 patients (17.5\% complication rate; 3 from the NF cohort and 5 from the PF cohort) that all resolved without clinical sequelae. There were 3 cases of superficial cellulitis (2 PF, 1 NFcohorts), 1 (NF cohort) deep-soft tissue wound infection, and 1 (PF cohort) large buttock hematoma. Very early in our experience, immediate postop CT scan revealed implant penetration into the sacral neural foramen in 2 patients (1 $\mathrm{NF}, 1 \mathrm{PF}$ cohort). Both patients were brought back to the OR and the implants were retracted to the edge of the neural foramen, resulting in complete resolution of symptoms.

In one patient (NF cohort) with an unrecognized hemisacralized L5 transitional vertebrae, the first implant was inadvertently placed too cephalad, compressing the L5 nerve. This patient was brought back to the OR for a partial retraction of the implant and experienced a complete resolution of symptoms. This same patient had a nondisplaced fracture located at the inferior edge of the Ilium adjacent to the sciatic notch at the edge of the lowest implant. The fracture healed without intervention. CT scans performed at 12 months on all 7 patients revealed evidence of bone ongrowth with no suggestion of implant loosening.

\section{DISCUSSION}

SI joint symptoms can present as pain in the SI joint, low back, hip, groin, or buttock.As shown by Sembrano and Polly, many patients have a combination of pain generators [10]. Additionally, a back pain patient's ability to identify discrete areas of pain may in part be influenced by the relatively small cortical region in the sensory homunculus responsible for the rather large lumbopelvic hip complex [10]. As a result, a careful and thorough clinical and physical exam must be performed to correctly identify the pain generator. Positive provocative maneuvers combined with $75 \%$ pain relief after image guided SI joint injection has

Table 1. Demographics

\begin{tabular}{|c|c|c|c|}
\hline & $\begin{array}{l}\text { NF: Patients with No History of Spinal Fusion } \\
\text { or Confounding Lumbar Pathology }\end{array}$ & $\begin{array}{c}\text { PF: Patients with History of Prior } \\
\text { Lumbar Spinal Fusion }\end{array}$ & $\begin{array}{l}\text { LP: Patients with Concomitant Lumbar } \\
\text { Pathology Treated Non-Surgically }\end{array}$ \\
\hline $\mathrm{N}$ & 18 & 15 & 7 \\
\hline Age (years) & $49( \pm 12)$ & $58( \pm 11)$ & $58( \pm 17)$ \\
\hline Sex & $\begin{array}{c}12(67 \%) \text { female } \\
6(33 \%) \text { male }\end{array}$ & $\begin{array}{c}11(73 \%) \text { female } \\
4(27 \%) \text { male }\end{array}$ & $\begin{array}{c}3(43 \%) \text { female } \\
4(57 \%) \text { male }\end{array}$ \\
\hline OR time (stdev) & $60( \pm 19) \mathrm{min}$ & $64( \pm 19) \mathrm{min}$ & $64( \pm 19) \mathrm{min}$ \\
\hline
\end{tabular}

Table 2. NRS Pain Scores Reported as Mean Change from Baseline (Standard Deviation) and Patient Satisfaction

\begin{tabular}{|c|c|c|c|c|c|}
\hline & 3mo NRS & 6mo NRS & 12mo NRS & 24mo NRS & 24mo Satisfaction \\
\hline \hline NF cohort $(\mathrm{n}=18)$ & $-4.53(2.68)$ & $-4.74(2.55)$ & $-5.94(3.30)$ & $-5.47(2.88)$ & $89 \%$ \\
\hline PF cohort $(\mathrm{n}=15)$ & $-3.47(2.74)$ & $-4.0(2.84)$ & $-3.50(3.46)$ & $-5.81(3.50)$ & $92 \%$ \\
\hline LP cohort $(\mathrm{n}=7)$ & $-3.5(3.07)$ & $-3.93(2.86)$ & $-3.71(3.11)$ & $-4.79(4.28)$ & $63 \%$ \\
\hline
\end{tabular}


been shown to be a reliable method for diagnosing the SI joint as the pain generator $[11,12]$.

Table 3. Type and Frequency of Prior Lumbar Spinal Fusion and Lumbar Pathology Managed Conservatively

\begin{tabular}{|c|c|}
\hline Type of Surgery & Frequency \\
\hline \multicolumn{2}{|l|}{ General frequency } \\
\hline Single level fusion & 4 \\
\hline Multi-level fusion & 11 \\
\hline Hip replacement surgery & 1 \\
\hline \multicolumn{2}{|l|}{ Specific frequency } \\
\hline L3-L5 & 1 \\
\hline $\mathrm{L} 4 / 5$ & 1 \\
\hline L3-S1 & 1 \\
\hline L4-S1 & 9 \\
\hline L5-S1 & 3 \\
\hline
\end{tabular}

While all 3 cohorts in our study improved, it is not surprising that the NF cohort in our study were younger and experienced a greater reduction in pain scores than the other two groups. Patients with a history of prior lumbar spinal fusion reported a slightly greater satisfaction with surgery. It is possible that these patients suffered from varying degrees of failed back surgery syndrome, again emphasizing the need to correctly identify the pain generators to the degree any single or combination of pain sources can be identified in such a complex population (low back pain). The complexity of the population and discrimination between pain sources is further illustrated by the lesser degree of satisfaction reported by patients who had no history of prior lumbar spine fusion, but who have known concomitant symptomatic spinal pathology treated with medical management. Though this cohort (LP) was smaller than the other 2, the results show a meaningful trend.

SI joint arthrodesis has been previously described as a treatment option for pain refractory to conservative care(1320]. Various methods are described in the literature. Buchowski et al. reported significant improvements in pain and function as measured by SF-36 on 20 patients who underwent SI joint arthrodesis using a posterior modified

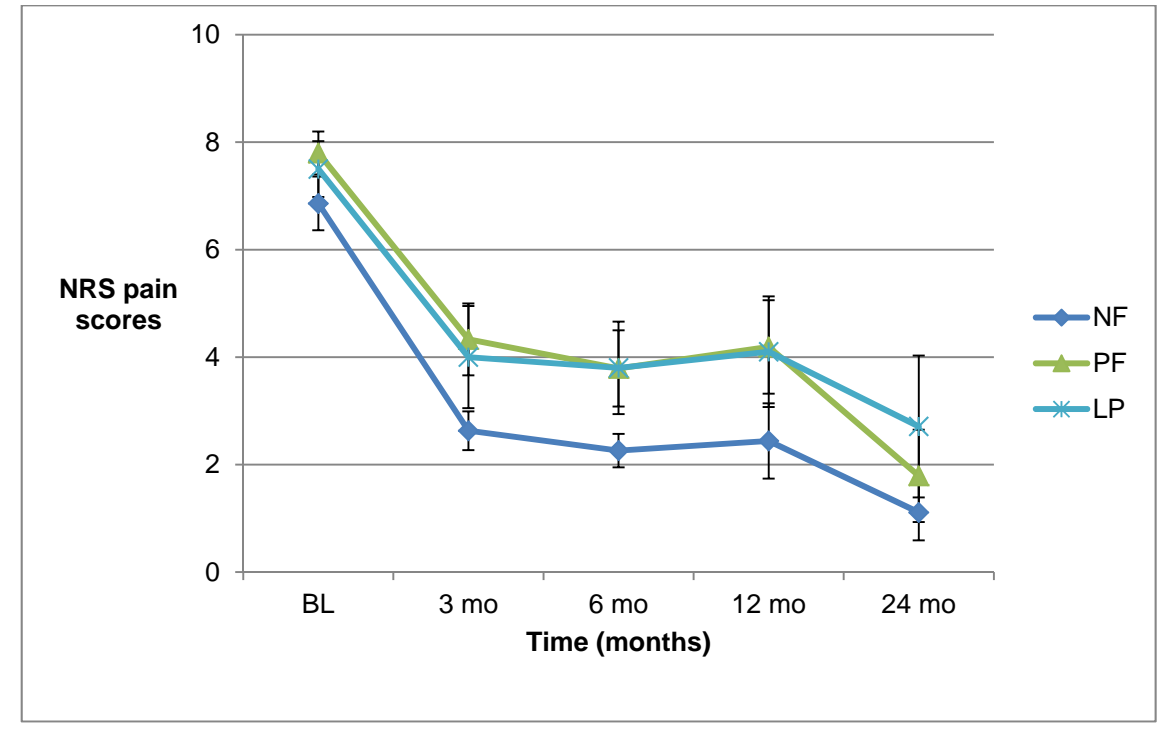

Fig. (2). NRS pain scores across groups at each time point.

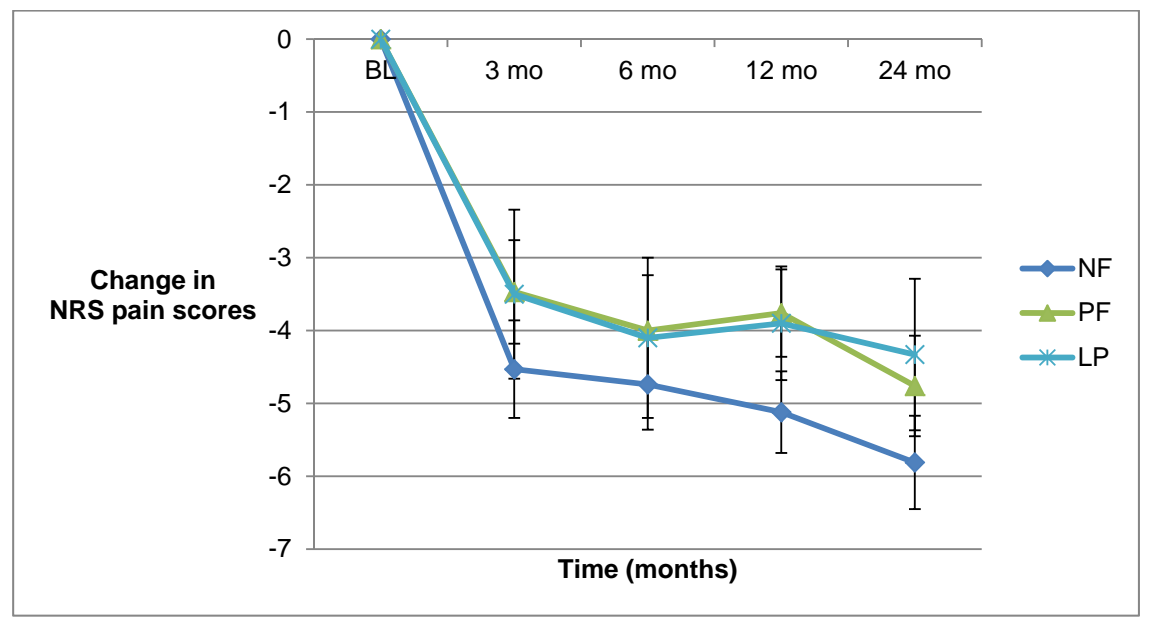

Fig. (3). Change in NRS scores across timepoints. 
Smith-Peterson technique [13]. The Smith Peterson technique involves excision of a $2.5 \times 3.5 \mathrm{~cm}$ iliac bone graft that is then secured within the SI joint using $6.5 \mathrm{~mm}$ AO bone screws. Buchowski et al. modified this technique to remove the bone window directly over the SI joint, exposing the articular surface of the joint. The joint surface was curetted prior to graft reinsertion and fixation with plates and screws. Reported complications included a $15 \%$ nonunion rate that required revision using an anterior approach and two associated deep wound infections. Although open surgery may result in positive pain and function improvements, the procedure is invasive and comes with a high revision rate.

Arthrodesis using a percutaneous approach with screws or threaded cages has been described with positive results. Al-Khayer et al. reported on 9 patients using a single hollow modular anchorage (HMA) screw packed with bone graft [17]. All patients experienced a clinically significant improvement in VAS pain scores and all but 1 patient improved in function. One patient suffered a deep wound infection. Khurana at al. also report on an HMA with demineralized bone matrix in a cohort of 15 patients with relatively good outcomes [19]. Wise and Dall reported good clinical results after fusing 13 patients with an $11 \times 25 \mathrm{~mm}$ threaded cage packed with rhBMP-2 [21]. The cage was inserted posteriorly within the joint rather than across the joint as reported in previous studies. Due to the off-label nature and elevated cost associated with rhBMP-2, autologous iliac crest harvest was suggested. However, studies suggest that this can lead to further degeneration of the SI joint. The various MIS methods described represent an improvement to the open surgical technique but leave room for improvement in technique and patient outcomes.

It is well known that minimally invasive methods for other surgical procedures have reduced peri-operative morbidity. Advantages of this reported MIS SI joint fusion implant technique include a small incision, minimal blood loss, bone and ligament preservation, and a relatively short period of immobilization.

Like all studies, this one has advantages and limitations. One advantage is the single surgeon experience. This allows for consistency in diagnosis and surgical technique. The study design is a limitation. As is common with medical chart reviews, some intermittent data points are missing as not all patients returned for follow up as instructed. A limitation to nearly all studies in spine is the assessment of pain. Like many of our colleagues, we use a numerical rating scale in our clinic as a convenient way of measuring progress across timepoints. This methodology, like all pain instruments is not perfect. Pain is a multi-dimensional experience not comprehensively captured on a unidimensional instrument such as NRS or VAS (visual analog scale).

\section{CONCLUSION}

Patients with SI joint pain, regardless of history of lumbar spinal fusion, show a clinically and statistically significant improvement in pain after minimally invasive SI joint fusion. Patients who have not undergone prior lumbar spinal fusion surgery are younger and experience greater improvement in pain scores. The presence of concomitant symptomatic lumbar spine pathology potentially confounds the treatment effect as patients may not be able to discriminate between symptoms arising from the SI joint and the lumbar spine. These patients tend to report lower satisfaction with surgery even though their pain scores improve clinically as well as statistically. Patients with a history or prior lumbar spinal fusion report greater satisfaction than the other two cohorts in our report.

\section{CONFLICT OF INTEREST}

No funds were received in support of this research. The author holds stock in and is a consultant for SI-BONE, Inc., the manufacturer of the implant used in this study.

\section{ACKNOWLEDGEMENTS}

The author wishes to acknowledge Robyn Capobianco for writing assistance and editorial review, and Daniel Cher for statistical analysis.

\section{REFERENCES}

[1] Weksler N, Velan GJ, Semionov M, et al. The role of sacroiliac joint dysfunction in the genesis of low back pain: the obvious is not always right. Arch Orthop Trauma Surg 2007; 127(10): 885-8.

[2] Foley BS, Buschbacher RM. Sacroiliac joint pain: anatomy, biomechanics, diagnosis, and treatment. Am J Phys Med Rehabil 2006; 85(12): 997-1006.

[3] Schwarzer AC, Aprill CN, Bogduk N. The sacroiliac joint in chronic low back pain. Spine 1995; 20(1): 31-7.

[4] Rudolf L. Sacroiliac joint arthrodesis - MIS technique with titanium implants: report of the first 50 patients and outcomes. Open Orthop J 2012; 6: 495-502.

[5] Copay AG, Glassman SD, Subach BR, Berven S, Schuler TC, Carreon LY. Minimum clinically important difference in lumbar spine surgery patients: a choice of methods using the Oswestry Disability Index, Medical Outcomes Study questionnaire Short Form 36, and pain scales. Spine J 2008; 8(6): 968-74.

[6] Ha K-Y, Lee J-S, Kim K-W. Degeneration of sacroiliac joint after instrumented lumbar or lumbosacral fusion: a prospective cohort study over five-year follow-up. Spine 2008; 33(11): 1192-8.

[7] DePalma MJ, Ketchum JM, Saullo TR. Etiology of Chronic Low Back Pain in Patients Having Undergone Lumbar Fusion. Pain Med [Internet]. 2011 Apr 11 [cited 2011 May 10]; Available from: http: //www.ncbi.nlm.nih.gov/pubmed/21481166

[8] Maigne JY, Planchon CA. Sacroiliac joint pain after lumbar fusion. A study with anesthetic blocks. Eur Spine J 2005; 14(7): 654-8.

[9] Maldjian C, Mesgarzadeh M, Tehranzadeh J. Diagnostic and therapeutic features of facet and sacroiliac joint injection. Anatomy, pathophysiology, and technique. Radiol Clin North Am 1998; 36(3): 497-508.

[10] Sembrano JN, Polly DW. How Often is Low Back Pain Not Coming From the Back?. Spine 2008 34(1): E27-32.

[11] Szadek KM, van der Wurff P, van Tulder MW, Zuurmond WW, Perez RSGM. Diagnostic validity of criteria for sacroiliac joint pain: a systematic review. J Pain 2009; 10(4): 354-68.

[12] Liliang P-C, Lu K, Liang C-L, Tsai Y-D, Wang K-W, Chen H-J. Sacroiliac joint pain after lumbar and lumbosacral fusion: findings using dual sacroiliac joint blocks. Pain Med 2011; 12(4): 565-70.

[13] Buchowski JM, Kebaish KM, Sinkov V, Cohen DB, Sieber AN, Kostuik JP. Functional and radiographic outcome of sacroiliac arthrodesis for the disorders of the sacroiliac joint. Spine J 2005; 5(5): 520-28; discussion 529.

[14] Giannikas KA, Khan AM, Karski MT, Maxwell HA. Sacroiliac joint fusion for chronic pain: a simple technique avoiding the use of metalwork. Eur Spine J 2004; 13(3): 253-6.

[15] Smith-Petersen MN. Arthrodesis of the sacroiliac joint. A new method of approach. J Bone Joint Surg Am 1921; 3(8): 400-5.

[16] Moore MR. Surgical treatment of chronic painful sacroiliac joint dysfunction. Movement, stability, and low back pain : the essential role of the pelvis. New York: Churchill Livingstone 1997; pp. 56372.

[17] Al-Khayer A, Hegarty J, Hahn D, Grevitt MP. Percutaneous sacroiliac joint arthrodesis: a novel technique. J Spinal Disord Tech 2008; 21(5): 359-63. 
[18] Belanger TA, Dall BE. Sacroiliac arthrodesis using a posterior midline fascial splitting approach and pedicle screw instrumentation: a new technique. J Spinal Disord 2001; 14(2): 118-24.

[19] Khurana A, Guha AR, Mohanty K, Ahuja S. Percutaneous fusion of the sacroiliac joint with hollow modular anchorage screws: clinical and radiological outcome. J Bone Joint Surg Br 2009; 91(5): 627-31.
[20] Güner G, Gürer S, Elmali N, Ertem K. Anterior sacroiliac fusion: a new video-assisted endoscopic technique. Surg Laparosc Endosc 1998; 8(3): 233-6.

[21] Wise CL, Dall BE. Minimally invasive sacroiliac arthrodesis: outcomes of a new technique. J Spinal Disord Tech 2008; 21(8): 579-84.

(C) Leonard Rudolf; Licensee Bentham Open

This is an open access article licensed under the terms of the Creative Commons Attribution Non-Commercial License (http://creativecommons.org/licenses/by-nc/3.0/) which permits unrestricted, non-commercial use, distribution and reproduction in any medium, provided the work is properly cited. 